\title{
Gène Cou nu et performances de croissance de poulets à deux saisons différentes en Egypte
}

\author{
A. ZEIN-EI-DEIN *, H. AYOUB * et P. MERAT ** \\ * Department of animal Production, Faculty of Agriculture, \\ Aïn-Shams University, Cairo, Egypt \\ ** I.N.R.A., Laboratoire de Génétique factorielle, \\ Centre de Recherches zootechniques \\ F 78350 Jouy-en-Josas
}

\begin{abstract}
Résumé
Dans deux croisements différents présentant une ségrégation au locus $N a$, l'un éclos en octobre 1978, l'autre en avril 1980, la croissance a été comparée en élevage au sol jusqu’à 12 semaines au Caire. La comparaison, dans le premier cas, portait sur les 3 génotypes $\mathrm{NaNa}$, Nana+ (cou nu) et na+na+ (plumage normal), et, dans le second cas, seulement sur les génotypes $\mathrm{Nana}+$ et $n a+n a+$. En automne 1978, les différences de croissance entre génotypes sont faibles, avec un léger avantage de poids pour les poulets à plumage normal à 4 et 8 semaines. Par contre, au printemps 1980 , à partir de 8 semaines et jusqu'à 12 semaines d'âge, les hétérozygotes "cou $n u$ » montrent un avantage pondéral sur les poulets à plumage normal de l'ordre de 11 p. 100 chez les mâles et 9 p. 100 chez les femelles. La différence importante des températures moyennes entre les deux saisons et le rapprochement avec des résultats antérieurs à température contrôlée suggèrent que l'avantage du génotype $\mathrm{Nana}+$ correspond à une meilleure thermotolérance en période de croissance.
\end{abstract}

\section{1. - Introduction}

Le gène $\mathrm{Na}$ (Cou nu) s'exprime dès la naissance par une réduction de l'extension du plumage dans certaines zones (HUTT, 1949), avec dominance incomplète (CRAWFORD, 1976 ; SCOTT \& CRAWFORD, 1977). En réponse à une température élevée constituant un «stress » thermique, SMITH \& LEE (1977) observent moins de mortalité parmi des poussins hétérozygotes $\mathrm{Na} \mathrm{na}^{+}$que pour ceux de génotype $\mathrm{na}^{+} \mathrm{na}^{+}$à plumage normal. D'autre part, BoRDAS et al. (1978), puis MONNET et al. (1979) ont montré qu'à une température ambiante élevée $\left(31^{\circ} \mathrm{C}\right)$ maintenue constante de 3 à 10 semaines d'âge par comparaison avec un témoin subissant des températures plus basses $\left(15\right.$ à $\left.20^{\circ} \mathrm{C}\right)$, la présence du gène $\mathrm{Na}$ s'accompagnait d'un avantage de croissance dans le groupe chauffé, avec un indice de consommation au moins aussi bon que celui des poulets à plumage normal. Les mêmes auteurs montrent en outre qu'aux deux températures, le rendement à l'abattage était amélioré par le gène «cou nu». 
Ces résultats suggéraient que, au moins dans des conditions de température analogues à celles réalisées, l'introduction du gène $N a$ dans des populations destinées à la production du poulet de chair pourrait être avantageuse. Cependant, cette conclusion demande à être vérifiée dans des conditions plus proches de celles d'une utilisation pratique éventuelle. C'est dans cet esprit qu'une première comparaison des génotypes au locus $N a$ a été faite en Egypte à deux saisons où les températures moyennes d'élevage étaient nettement différentes.

\section{2. - Matériel et méthodes}

Le présent travail a été entrepris dans le cadre d'une coopáration entre le Département de Production animale, Faculté d'Agriculture, Université Aïn-Shams, Le Caire, Egypte, et le Laboratoire de Génétique Factorielle, C.N.R.Z., I.N.R.A., Jouyen-Josas, France.

\section{1. - Matériel animal}

Deux séries d'éclosions ont été réalisées, chacune à une saison distincte, et à partir de deux groupes de reproducteurs différents.

\section{a) Le 25 octobre 1978}

Des poussins issus d'une partie du troupeau expérimental du Laboratoire de Génétique factorielle, provenant de 10 pères et 70 mères, sont éclos et ont été envoyés au Caire. Les parents des deux sexes étaient hétérozygotes $\mathrm{Nana}$. En conséquence, les poussins éclos (515 au total) se répartissaient entre les trois génotypes $\mathrm{Na} \mathrm{Na}$ (homozygotes $\mathrm{cou} n u$ ), $\mathrm{Nana} a^{+}$(hétérozygotes cou $n u$ ) et $n a^{+} n a^{+}$(plumage normal).

\section{b) Au printemps de 1980}

7 coqs hétérozygotes $\mathrm{Nana^{+ }}$, descendants d'animaux appartenant à l'éclosion précédente, ont été croisés avec 54 poules Fayoumi (origine : El-Fayoum Poultry Research Station, Agricultural Research Centre, Ministry of Agriculture, Egypt). Trois éclosions successives ont été obtenues, les 5, 12 et 19 avril. Les taux d'éclosion (poussins nés/œufs incubés) étaient respectivement : 77,8; 71,7 et 57,9 p. 100 pour les trois lots successifs, soit 69,1 p. 100 pour l'ensemble des trois éclosions. Les mères étant toutes à plumage normal $\left(n a^{+} n a^{+}\right)$, la descendance comportait les deux génotypes $\mathrm{Nana}$ (cou nu hétérozygote) et $n a^{+} n a^{+}$.

\section{2. - Conditions expérimentales}

Tous les poussins des deux séries (a et b) étaient démarrés en batterie chauffée électriquement jusqu'à l'âge de 4 semaines. De 4 à 12 semaines, ils étaient élevés 
en cellules au sol, sans chauffage le jour, mais avec, de 18 heures à 6 heures du matin environ, un chauffage d'appoint par lampe infra-rouge pour éviter une baisse trop importante des températures nocturnes.

L'aliment (à 18 p. 100 de protéines totales et $2800 \mathrm{Kcal} / \mathrm{kg} \mathrm{E.M.)} \mathrm{et} \mathrm{l'eau}$ étaient distribués ad libitum. La durée journalière d'éclairement était de 24 heures pendant la première semaine d'âge et de 14 heures ensuite jusqu'à 12 semaines.

Compte tenu de la saison respective d'élevage des deux séries a (éclose en octobre 1978) et b (éclose en avril 1980) la température ambiante, au moins après 4 semaines d'âge, différait de façon importante entre ces deux groupes. Ceci est reflété par les relevés de température qui ont été faits au cours des deux saisons d'élevage. Ces relevés n'étaient pas continus, mais comportaient deux mesures journalières, l'une, proche du maximum, faite au milieu de la journée (vers 14 heures), l'autre, s'approchant du minimum, se situant, dans la première saison, le soir vers 18 heures avant la mise en route des chauffages d'appoint, et, dans la seconde saison, vers 6 heures du matin.

\section{Tableau 1}

Maxima et minima journaliers de température en locaux d'élevage de la $5^{\circ}$ à la $12^{\circ}$ semaine d'âge

Daily maxima and minima of ambient temperature in chick houses from the 5th to the 12th week of age

\begin{tabular}{|c|c|c|c|c|c|c|c|c|}
\hline \multirow{2}{*}{$\begin{array}{c}\text { Saison } \\
\text { et date d'éclosion }\end{array}$} & \multicolumn{8}{|c|}{ Températures $\left({ }^{\circ} \mathrm{C}\right)$ par semaines } \\
\hline & $\begin{array}{c}5^{\circ} \\
\text { sem. }\end{array}$ & $\begin{array}{c}6^{e} \\
\text { sem. }\end{array}$ & $\begin{array}{c}7^{\circ} \\
\text { sem. }\end{array}$ & $\begin{array}{c}8^{e} \\
\text { sem. }\end{array}$ & $\begin{array}{c}9^{\circ} \\
\text { sem. }\end{array}$ & $\begin{array}{l}10^{e} \\
\text { sem. }\end{array}$ & $\begin{array}{l}11^{\circ} \\
\text { sem. }\end{array}$ & $\begin{array}{l}12^{\circ} \\
\text { sem. }\end{array}$ \\
\hline \multicolumn{9}{|l|}{ 1. Automne 1978} \\
\hline \multicolumn{9}{|l|}{ Eclos. $25 / 10$} \\
\hline Maxima .. & 22,9 & 20,9 & 19,3 & 21,4 & 20,4 & 20,6 & 17,4 & 20,3 \\
\hline Minima...... & 10,4 & 12,9 & 11,6 & 11,4 & 12,1 & 9,0 & 7,0 & 9,9 \\
\hline \multicolumn{9}{|l|}{ 2. Printemps 1980} \\
\hline \multicolumn{9}{|l|}{ Eclos. $5 / 4$} \\
\hline Maxima & 31,9 & 29,9 & 33,3 & 36,0 & 35,0 & 34,0 & 35,3 & 35,4 \\
\hline Minima & 25,3 & 24,3 & 26,7 & 26,0 & 29,7 & 28,3 & 30,0 & 31,3 \\
\hline \multicolumn{9}{|l|}{ Eclos. $12 / 4$} \\
\hline Maxima & 29,9 & 33,3 & 36,0 & 35,0 & 34,0 & 35,3 & 35,4 & 38,0 \\
\hline Minima & 24,3 & 26,7 & 26,0 & 29,7 & 28,3 & 30,0 & 31,3 & 33,3 \\
\hline \multicolumn{9}{|l|}{ Eclos. $19 / 4$} \\
\hline Maxima & 33,3 & 36,0 & 35,0 & 34,0 & 35,3 & 35,4 & 38,0 & 35,6 \\
\hline Minima . & 26,7 & 26,0 & 29,7 & 28,3 & 30,0 & 31,3 & 33,3 & 30,7 \\
\hline
\end{tabular}


Dans les deux séries, la température était approximativement la même en ce qui concerne la phase d'élevage en batterie chauffée. Elle se situait entre 32 et $34{ }^{\circ} \mathrm{C}$ pour la première semaine, et entre 26 et $30^{\circ} \mathrm{C}$ pour les trois semaines suivantes. C'est dans la phase d'élevage au sol, de 4 à 12 semaines d'âge, que se situaient les différences liées aux conditions climatiques saisonnières. Le tableau 1 donne respectivement pour le lot éclos en octobre 1978 et pour les trois lots éclos en avril 1980 les maxima et minima absolus journaliers relevés dans les locaux d'élevage de la $5^{\prime \prime}$ à la $12^{\text {e }}$ semaine d'âge.

\section{3. - Critères mesurés; analyse statistique des données}

Les critères mesurés dans les deux séries expérimentales sont le poids corporel à l'âge de 1 jour, puis de 2 en 2 semaines jusqu'à 12 semaines, et la mortalité en cours d'élevage.

Pour le poids corporel à chaque âge, une analyse de variance est présentée avec les facteurs contrôlés «génotype» et «sexe»pour l'automne 1978. Au printemps 1980, une analyse a été faite séparément dans chaque sexe, avec les facteurs «génotype» et «date d'éclosion».

Le pourcentage de mortalité (0-12 semaines) est comparé entre génntypes par un test de $\chi^{2}$.

\section{3. - Résultats}

\section{1. - Mortalité}

La mortalité totale de 0 à 12 semaines ne différait nulle part suivant le sexe. Dans la première série (éclosion en octobre 1978), elle était respectivement, pour les génotypes $\mathrm{NaNa}, \mathrm{Nana}{ }^{+}$et $n a^{+} n a^{+}$, égale à 23,3 p. $100,22,6 \mathrm{p} .100$ et 23,1 p. 100 (différences non significatives : $\chi^{2}=0,23$ pour 2 d.l.). Pour l'ensemble des trois lots éclos en avril 1980, les pourcentages correspondants, respectivement pour les génotypes $\mathrm{Nana} a^{+}$et $n a^{+} n a^{+}$, étaient 26,8 et 27,3 p. $100\left(\chi^{2}=0,00\right.$ pour 1 d.1., non significatif). Cette mortalité relativement élevée se situait presque toute dans les 2 à 3 premières semaines d'âge. Elle a pu correspondre à des refroidissements nocturnes, aggravés au printemps 1980 par des coupures de courant arrêtant le chauffage des batteries.

\section{2. - Croissance pondérale}

Les tableaux 2 et 3 indiquent respectivement, pour les poussins éclos en octobre 1978, les poids corporels moyens aux divers âges par sexe et génotype, et les analyses de variance correspondantes. 
TABLEaU 2

Poids moyen par âge, sexe et génotype au locus $\mathrm{Na}$ : lot éclos en octobre 1978

Mean body weight according to age, sex and genotype at $\mathrm{Na}$ locus : group hatched in october 1978

\begin{tabular}{|c|c|c|c|c|c|c|c|}
\hline \multirow{3}{*}{\multicolumn{2}{|c|}{ Age et sexe }} & \multicolumn{6}{|c|}{ Génotype } \\
\hline & & \multicolumn{2}{|r|}{$\mathrm{Na} \mathrm{Na}$} & \multicolumn{2}{|r|}{$\mathrm{Na}$ na +} & \multicolumn{2}{|r|}{ nat nat } \\
\hline & & $\mathrm{N}$ & $\begin{array}{l}\text { Poids moyen } \\
\text { (g) }\end{array}$ & $\mathrm{N}$ & $\begin{array}{l}\text { Poids moyen } \\
(\mathrm{g})\end{array}$ & $\mathbf{N}$ & $\begin{array}{l}\text { Poids moyen } \\
(\mathrm{g})\end{array}$ \\
\hline \multicolumn{8}{|c|}{1 jour } \\
\hline$\hat{\sigma}$ & $\ldots \ldots$ & 75 & 41,0 & 134 & 41,7 & 55 & 42,2 \\
\hline 오 & $\ldots$ & 58 & 40,0 & 131 & 40,1 & 62 & 40,9 \\
\hline \multicolumn{8}{|c|}{2 semaines } \\
\hline$\hat{o}$ & $\ldots \ldots \ldots \ldots$ & 73 & 128,4 & 124 & 139,7 & 51 & 132,7 \\
\hline q & ........... & 56 & 125,4 & 104 & 130,2 & 60 & 131,8 \\
\hline \multicolumn{8}{|c|}{4 semaines } \\
\hline$\hat{o}$ & .. & 64 & 377,3 & 123 & 410,6 & 45 & 401,1 \\
\hline 웅 & $\ldots$ & 51 & 369,2 & 100 & 379,7 & 58 & 398,0 \\
\hline \multicolumn{8}{|c|}{6 semaines } \\
\hline$\hat{o}$ & & 65 & 803,8 & 122 & 837,1 & 46 & 8.55 .1 \\
\hline q & $\ldots \ldots \ldots \ldots$ & 52 & 735,0 & 100 & 733,9 & 57 & 770,2 \\
\hline \multicolumn{8}{|c|}{8 semaines } \\
\hline$\hat{\delta}$ & $\ldots \ldots$ & 65 & 1323,2 & 122 & 1350.2 & 46 & 13880 \\
\hline q & .......... & 52 & 1146,6 & 100 & 1079,4 & 58 & 1179,6 \\
\hline \multicolumn{8}{|c|}{10 semaines } \\
\hline$\hat{o}$ & $\ldots$ & 61 & 1817,2 & 114 & 1856,0 & 45 & 1904.2 \\
\hline 우 & .. & 52 & 1536,5 & 96 & 1500,8 & 57 & 1540.0 \\
\hline \multicolumn{8}{|c|}{12 semaines } \\
\hline$\hat{o}$ & & 53 & 2193,5 & 109 & 2280.0 & 34 & 2300.1 \\
\hline$q$ & & 49 & 1790,7 & 96 & 1779,6 & 56 & 1851,6 \\
\hline
\end{tabular}


TABleaU 3

Analyse de variance du poids corporel pour chaque âge : lot éclos en octobre 1978

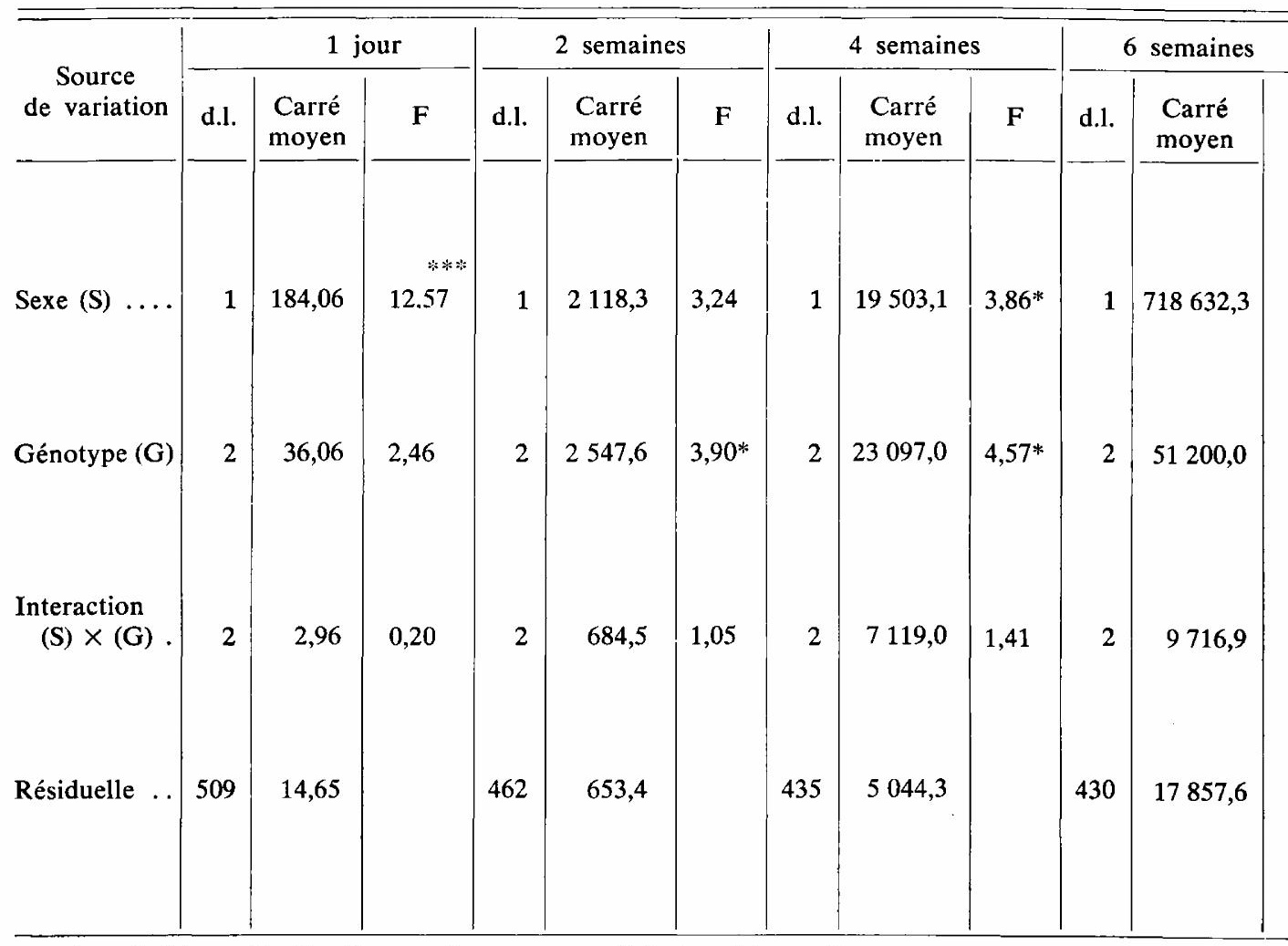

$(*)(* *)(* * *))$ : Significatif respectivement au seuil 5,1 et 0,1 p. 100 .

Le tableau 4 présente les poids moyens par âge et sexe pour les deux génotypes $\mathrm{Nana}{ }^{+}$et $\mathrm{na}^{+} \mathrm{na} \mathrm{a}^{+}$, pour les trois lots éclos en avril 1980 regroupés.

Le tableau 5 donne, également pour l'année 1980, les analyses de variance intra-sexe pour les poids à chaque âge, avec les facteurs contrôlés "génotype » (au locus $N a$ ) et «éclosion».

\section{4. - Discussion et conclusions}

Les génotypes ne présentant pas de différences pour la mortalité, nous ne ferons pas d'autres commentaires sur ce caractère. 
Analysis of variance of body weight at each age : group hatched in october 1978

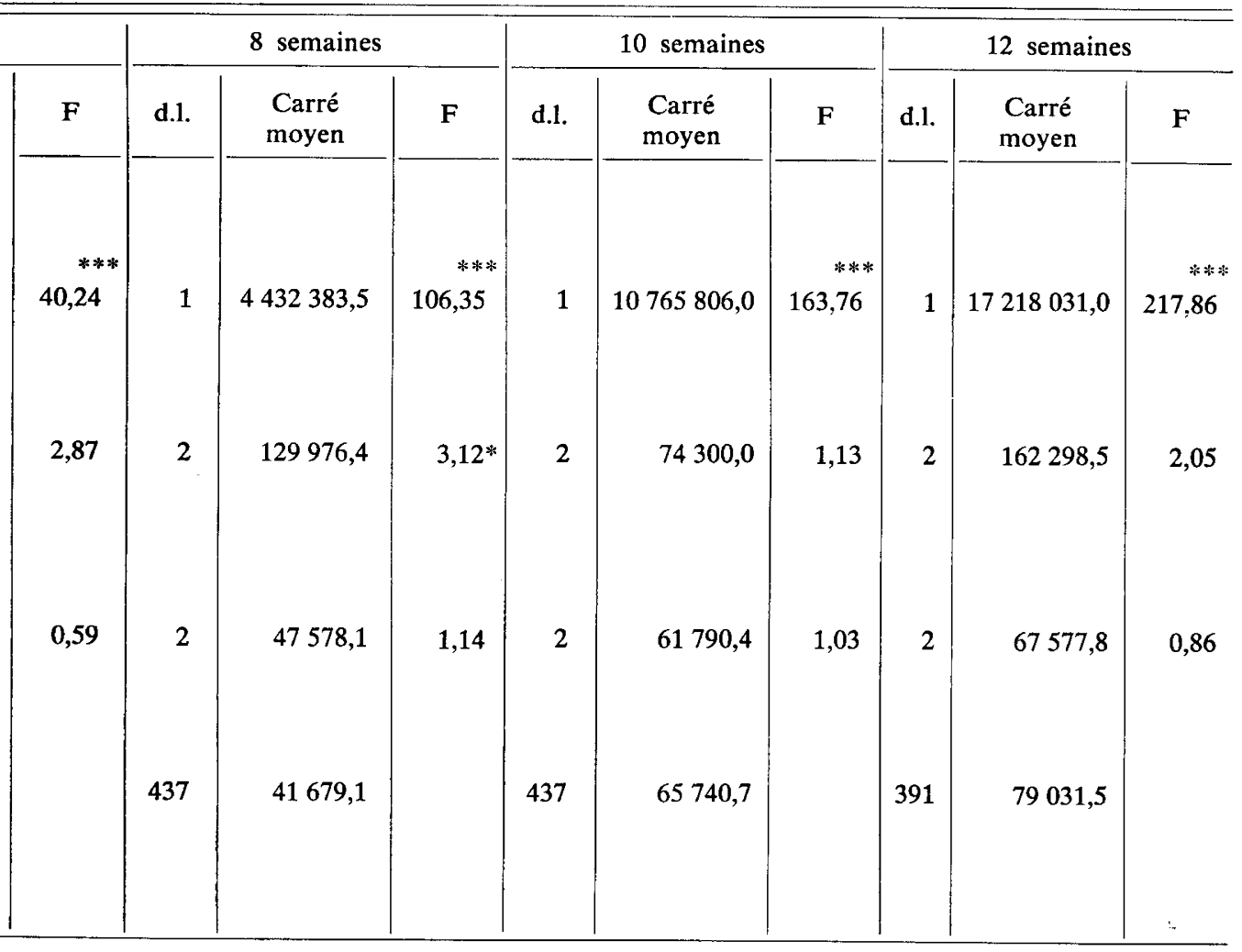

Quant aux mesures de croissance pondérale, nous présentons leur analyse et leur interprétation de façon séparée pour les données de 1978 et pour celles de 1980 , car ces deux séries expérimentales diffèrent non seulement par l'environnement saisonnier mais par le génotype moyen et les performances des oiseaux. Le croisement réalisé en 1980 avec la race Fayoumi était de plus petite taille à âge égal.

A l'automne 1978, un effet significatif à 5 p. 100 associé au génotype apparaît pour le poids corporel à 2,4 et 8 semaines (tableau 3), mais non aux autres âges. Il s'agit d'après l'examen des moyennes (tableau 2) d'un léger retard de croissance associé à la présence de l'allèle $N a$. Une partie de la différence peut d'ailleurs être rapportée à la réduction du poids du plumage chez les oiseaux «cou nu» (MonNET et al., 1979). On peut évaluer son ordre de grandeur à 8 semaines, à $24 \mathrm{~g}$ chez les mâles $\mathrm{Nana}{ }^{+}$et $20 \mathrm{~g}$ chez les femelles $\mathrm{Nana}{ }^{+}$, à $34 \mathrm{~g}$ chez les mâles $\mathrm{NaNa}$ et $30 \mathrm{~g}$ chez les femelles $\mathrm{NaNa}$. 


\section{TABLeaU 4}

Poids moyen par âge, sexe et génotype au locus $\mathrm{Na}$ : total des 3 lots éclos en avril 1980 Mean body weight according to age, sex and genotype at $\mathrm{Na}$ locus :

Total of the 3 hatches, April 1980

\begin{tabular}{|c|c|c|c|c|c|c|}
\hline \multirow{3}{*}{\multicolumn{2}{|c|}{ Age et sexe }} & \multicolumn{4}{|c|}{ Génotype } & \multirow{3}{*}{$\begin{array}{c}\mathrm{Nanat} \\
\text { en p. cent } \\
\text { de nat nat }\end{array}$} \\
\hline & & \multicolumn{2}{|c|}{$\mathrm{Nana}+$} & \multicolumn{2}{|c|}{$\mathrm{na}+\mathrm{na}+$} & \\
\hline & & $\mathbf{N}$ & $\begin{array}{l}\text { Poids moyen } \\
\text { (g) }\end{array}$ & $\mathbf{N}$ & $\begin{array}{l}\text { Poids moyen } \\
\text { (g) }\end{array}$ & \\
\hline \multicolumn{7}{|c|}{1 jour } \\
\hline$\hat{\sigma}$ & $\ldots \ldots \ldots \ldots$ & 88 & 31,0 & 90 & 30,3 & 102,3 \\
\hline$q$ & $\ldots \ldots \ldots \ldots$ & 106 & 29,5 & 93 & 28,9 & 102,1 \\
\hline \multicolumn{7}{|c|}{2 semaines } \\
\hline$\hat{\jmath}$ & $\ldots \ldots \ldots \ldots$ & 67 & 85,1 & 69 & 83,4 & 102,0 \\
\hline$\stackrel{9}{9}$ & $\ldots \ldots \ldots \ldots$ & 83 & 73,0 & 71 & 72,3 & 101,0 \\
\hline \multicolumn{7}{|c|}{4 semaines } \\
\hline$\hat{\sigma}$ & . & 65 & 193,5 & 66 & 188,3 & 102,8 \\
\hline$q$ & $\ldots \ldots \ldots \ldots$ & 81 & 164,9 & 70 & 162,9 & 101,2 \\
\hline \multicolumn{7}{|c|}{6 semaines } \\
\hline$\hat{o}$ & $\ldots \ldots$ & 64 & 388,5 & 66 & 355,9 & 109,2 \\
\hline$q$ & $\ldots \ldots \ldots \ldots$ & 81 & 318,2 & 70 & 303,9 & 104,7 \\
\hline \multicolumn{7}{|c|}{8 semaines } \\
\hline$\hat{\delta}$ & $\ldots$ & 64 & 555,5 & 63 & 498,3 & 111,5 \\
\hline 운 & $\ldots \ldots \ldots$ & 79 & 449,6 & 70 & 412,6 & 109,0 \\
\hline \multicolumn{7}{|c|}{10 semaines } \\
\hline$\hat{o}$ & $\ldots \ldots \ldots \ldots$ & 64 & 748,9 & 63 & 672,3 & 111,4 \\
\hline 우 & $\ldots \ldots \ldots \ldots$ & 79 & 601,5 & 70 & 551,1 & 109,1 \\
\hline \multicolumn{7}{|c|}{12 semaines } \\
\hline$\hat{\sigma}$ & $\ldots \ldots \ldots \ldots$ & 64 & 1013,3 & 63 & 905,2 & 111,9 \\
\hline q & $\ldots \ldots \ldots \ldots$ & 78 & 803,5 & 70 & 736,9 & 109,0 \\
\hline
\end{tabular}




\section{Tableau 5}

Analyse de variance du poids corporel pour chaque âge et par sexe : lots éclos en avril 1980

Analysis of variance for body weight at each age and for each sex : chicks hatched in April 1980

\begin{tabular}{|c|c|c|c|c|c|c|c|}
\hline \multirow{2}{*}{ Age } & \multirow{2}{*}{$\begin{array}{c}\text { Source } \\
\text { de variation }\end{array}$} & \multicolumn{3}{|c|}{ Mâles } & \multicolumn{3}{|c|}{ Femelles } \\
\hline & & d.l. & Variance & F & d.l. & Variance & $\mathbf{F}$ \\
\hline \multirow{4}{*}{1 jour } & Génotype & 1 & 11,29 & 3,03 & 1 & 19,14 & $6,19 *$ \\
\hline & Eclosion ..... & 2 & 23,51 & $6,29 * *$ & 2 & 46,03 & $14,90^{* *}$ \\
\hline & Interaction $\ldots$ & 2 & 0,47 & 0,12 & 2 & 3,12 & 1,01 \\
\hline & Résiduelle .... & 172 & 3,74 & & 193 & 3,09 & \\
\hline \multirow[t]{4}{*}{2 sem. } & Génotype & 1 & 134,07 & 1,69 & 1 & 7,49 & 0,16 \\
\hline & Eclosion ..... & 2 & 6,63 & 0,08 & 2 & 20,88 & 0,46 \\
\hline & Interaction $\ldots$ & 2 & 72,94 & 0,92 & 2 & 21,35 & 0,47 \\
\hline & Résiduelle .... & 130 & 79,46 & & 148 & 45,71 & \\
\hline \multirow[t]{4}{*}{4 sem. } & Génotype & 1 & 759,37 & $5,53^{*}$ & 1 & 273,66 & 2,53 \\
\hline & Eclosion. & 2 & 337,77 & 2,46 & 2 & 611,93 & $5,65 * *$ \\
\hline & Interaction & 2 & 54,04 & 0,39 & 2 & 23,48 & 0,22 \\
\hline & Résiduelle & 125 & 137,21 & & 145 & 108,38 & \\
\hline \multirow{4}{*}{6 sem. } & Génotype & 1 & 34734,62 & $59,61 * * *$ & 1 & 7965,42 & $19,47 * * *$ \\
\hline & Eclosion. & 2 & 1032,43 & 1,79 & 2 & 1236,15 & 3,02 \\
\hline & Interaction & 2 & 410,58 & 0,71 & 2 & 201,66 & 0,49 \\
\hline & Résiduelle & 124 & 576,60 & & 145 & 409,07 & \\
\hline \multirow[t]{4}{*}{8 sem. } & Génotype & 1 & 103099,91 & $90,12 * * *$ & 1 & 50176,82 & $63,20^{* * *}$ \\
\hline & Eclosion . & 2 & 1110,55 & 0,97 & 2 & 1744,17 & 2,20 \\
\hline & Interaction & 2 & 714,44 & 0,62 & 2 & 606,46 & \\
\hline & Résiduelle & 121 & 1144,00 & & 143 & 793,98 & \\
\hline \multirow[t]{4}{*}{10 sem. } & Génotype & 1 & 185387,51 & $80,40 * * *$ & 1 & 92593,57 & $59,54 * * *$ \\
\hline & Eclosion . & 2 & 1613,20 & 0,70 & 2 & 2720,96 & 1,75 \\
\hline & Interaction & 2 & 1968,78 & 0,85 & 2 & 1461,62 & 0,94 \\
\hline & Résiduelle & 121 & 2305,79 & & 143 & 1555,22 & \\
\hline \multirow[t]{4}{*}{12 sem. } & Génotype & 1 & 371163,60 & & 1 & 159860,30 & $57,70^{* * *}$ \\
\hline & Eclosion . & 2 & 3734,45 & 0,89 & 2 & 4661,98 & 1,68 \\
\hline & Interaction & 2 & 6108,29 & 1,46 & 2 & 2282,85 & 0,82 \\
\hline & Résiduelle .. & 121 & 4187,31 & & 142 & 2770,47 & \\
\hline
\end{tabular}

$\left(^{*}\right)(* *)(* * *)$ Significatif respectivement au seuil 5,1 et 0,1 p. 100. 
Au printemps 1980, par contre, le tableau 5 fait ressortir une différence entre génotypes $\mathrm{Nana} a^{+}$et $\mathrm{na}^{+} \mathrm{na}^{+}$. Cette différence est faible jusqu'à 4 semaines et le fait qu'elle soit significative à 5 p. 100 chez les femelles à 1 jour est difficile à interpréter. Par contre, elle devient très hautement significative dans les deux sexes à partir de l'âge de 6 semaines, marquant une nette supériorité du génotype «cou nu » hétérozygote jusqu'à 12 semaines, et ceci en dépit, cette fois, du plumage moins abondant dans ce génotype. L'avantage relatif de poids vif des poulets mâles «cou $n u$ » se maintient entre 11 et 12 p. 100 de 8 à 12 semaines, alors que l'avantage correspondant chez les femelles du même génotype reste voisin de 9 p. 100.

Quoique, comme il a été déjà signalé, la comparaison de l'automne 1978 et celle du printemps 1980 portent sur des populations aux performances de croissance très différentes, et que d'autre part la première expérience inclut les trois génotypes au locus $N a$ et la seconde deux seulement, il nous a paru intéressant de rapprocher, pour leur interprétation, ces résultats entre eux et avec ceux de BoRDAs et al. (1978) et de Monnet et al. (1979). En effet, les températures subies par le lot éclos le 25-10-1978 sont, dans l'ensemble, modérées, alors que, pour les lots nés en avril 1980, la température moyenne est beaucoup plus élevée, avec des maxima journaliers dépassant généralement $30^{\circ} \mathrm{C}$. Ce n'est que dans ces conditions que les animaux "cou $n u$ » manifestent un avantage pour la croissance. Il en était de même pour les lots «chauffés» de Bordas et al. (1978) et MonNET et al. (1979), par opposition aux lots "témoins》 des mêmes expériences dont le régime de température était relativement comparable à celui de l'automne-hiver 1978 au Caire. Le présent résultat suggère donc que, dans les conditions d'élevage réalisées à l'Université Aïn-Shams et en saison chaude, le gène «cou $n u$ » apporte un gain appréciable de croissance, vraisemblablement par son effet favorable à la thermorégulation par accroissement de la thermolyse. Des données complémentaires seront souhaitables pour savoir si cet effet peut avoir une portée pratique dans les conditions de l'Egypte. En particulier, il sera nécessaire de vérifier, si, comme dans le résultat de MoNNET et al. (1979) à température élevée, l'amélioration de croissance produite par le gène $\mathrm{Na}$ s'opère sans détérioration de l'indice de consommation.

Reçu pour publication en juillet 1981.

\section{Remerciements}

Nous remercions le Dr R.D. Crawford (University of Saskatchewan, Saskatoon, Canada) et le Dr J.C. Blum (Station de Recherches avicoles, 37 - Nouzilly) de leurs remarques et suggestions constructives à la lecture de ce manuscrit.

\section{Summary}

Naked neck gene and growth performance of chicks at two seasons in Egypt

In two different crosses segregating at $\mathrm{Na}$ locus, one hatched in octobre 1978, the other in april 1980, growth was compared in floor pens till 12 weeks of age in Cairo. The comparison included in the first case the three genotypes $\mathrm{NaNa}, \mathrm{Na}$ nat (naked neck) and 
$n a+n a+$ (normal plumage). In the second case, only $\mathrm{Nana+}$ and $n a+n a+$ genotypes were available. In autumn 1978, growth differences between genotypes showed only a slight advantage at 4 and 8 weeks for chicks with normal plumage. On the contrary, in spring 1980 from 8 weeks to 12 weeks of age, naked neck heterozygotes compared to nat na+ chicks show a body weight about 11 p. 100 superior in males and 9 p. 100 in females. The large difference in mean ambient temperatures between the two seasons as well as the apparent similarity with former results obtained at controlled temperature suggest that this advantage of the $\mathrm{Nana}+$ genotype corresponds to a better heat tolerance during the growth period.

\section{Références bibliographiques}

Bordas A., Merat P., Sergent D., Ricard F.H., 1978. Influence du gène $\mathrm{Na}$ («Cou $n u »)$ sur la croissance, la consommation alimentaire et la composition corporelle du Poulet selon la température ambiante. Ann. Génét. Sél. anim., 10, 209-231.

CraWford R.D., 1976. Incomplete dominance of the gene for naked neck in the domestic fowl. Poult. Sci., 55, 820-822.

HutT F.B., 1949. Genetics of the fowl. McGraw-Hill, New York.

Monnet L.E., Bordas A., Merat.P., 19.79. Gène cou nu et performances de croissance selon la température chez le Poulet. Ann. Génét. Sél. anim., 11, 397-412.

Scott T., Crawford R.D., 1977. Feather number and distribution in the throat tuft of naked neck chickens. Poult. Sci., 56, 686-688.

Smith T., Lee R., 1977. A study of the naked neck gene of the fowi. Pouli. Sci., 56. 1758 (abstr.). 